\title{
PEDOT Chain Length and Excitation Energy Dependence from Time-Dependent Density Functional Theory
}

Ageo M. de Andrade, Luis H. Lacerda, Renan A. P. Ribeiro, Sergio R. de Lazaro \& Alexandre C. Junior

\section{Introduction}

Nowadays, the need to produce alternative energy sources which can substitute fossil fuels is a worldwide challenge. Furthermore, policy efforts must be made to become the clean energy technologies competitive to cheaper energy sources, and its application in the society, especially for reducing global warming ${ }^{1}$. Since 1970 , solar photovoltaic devices has been developed with objective to reach new low energy cost devices, but its application was limited by low energy conversion efficiency. During last decades, solar energy conversion up to $30 \%$ was achieved in inorganic devices based on crystalline silicon devices; however, they are limited by high cost and short durability ${ }^{2}$.

One way to avoid the high effective cost of solar cell devices is to replace silicon by organic semiconductors (OS) because its potential for easy roll-to-roll fabrication has more processable advantages mainly, high flexibility, easy to synthesize and endless possibilities on discovering new materials by organic modification, and capacity to use in flexible substrates ${ }^{3}$. However, such OS also have the problem of the low efficiency at energy conversion ${ }^{4}$. Recently, studies with multiple organic solar cells called "sandwich" solar cells have shown good candidates to avoid the limited absorption range ${ }^{5,6}$. Furthermore, OS can be associated with inorganic semiconductors to produce flexible thin film devices with good charge transport properties?. The role of organic materials that can be used for application in solar cell devices is widespread in different organic classes, but the main characteristic found in all compounds is $\pi$ conjugation length allowing a high charge mobility between HOMO and

\section{LUMO orbitals ${ }^{8,9}$.}

Organic polymeric materials used in solar cell devices must show high structure ordering of polymer bulk, once this molecular property contribute significantly for charge transport between HOMOLUMO orbitals spread in polymeric chain ${ }^{8,10,11}$. For better bulk formation efficiency, polymeric materials must show planar chain among monomers to produce charge carriers on polymeric chain ${ }^{12}$. Considering such property, the class of polythiophenes (PT) have attached attention, especially by experimental results in different synthesis routes ${ }^{13,14}$. From many PT derivates, Poly(3,4ethylenedioxythiophene) (PEDOT - Chart 1) has been investigated since 1970, initially on BAYER laboratories, become it an interesting candidate for application as OS, once PEDOT is easy to process in non-aqueous solvents, and it has excellent hole transport properties, allowing the application on different electronic devices, such as antistatic coatings, cathodes in capacitors, organic light emitting diodes (OLEDs), photovoltaic, and electrochromic fims ${ }^{15,16}$.

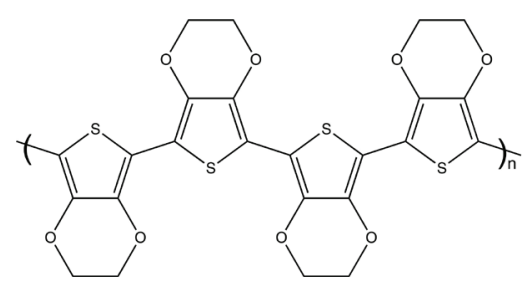

Chart 1. Chemical structure of Poly(3,4-ethylenedioxythiophene) (PEDOT) 
PEDOT has been investigated to understand the contribution of $\mathrm{p}$ conjugations on optical properties. Apperloo and co-workers ${ }^{17}$ investigated the relation between monomeric units and ultraviolet absorption through experimental and semi-empirical results, but from this contribution there is no significant theoretical contributions for a better understanding of the relation between molecular geometry, electronic structure and absorption spectra.

In this work we present a Time-Dependent Density Functional Theory (TD-DFT) investigation from monomer to sixamer PEDOT to deep on optical property, molecular geometry, $\pi$ conjugation length, electronic properties (HOMO and LUMO) and excitation profiles. The discussion is based on theoretical results of excitation levels on each oligomeric size comparing the differences between each geometry from molecular orbital and electronic property.

\section{Computacional Procedure}

3,4-ethylenedioxythiophene (EDOT) structures from monomer to decamer (1-EDOT to 10-EDOT) were optimized using PM618 semi-empirical methodology available in Molecular Package 2012 (MOPAC2012) Program ${ }^{19}$, where the gradient minimization was specified as 0.01 kcal.mol1 applying Pulay and Camp ${ }^{20}$ routines for SCF calculation. From optimized geometries, single-point calculation using GAMESS ${ }^{21-23}$ was applied using B3LYP/6-31+G(d,p) level of theory, with geometry gradient convergence tolerance of $1 \times 10-6$ Hartree, and SCF density convergence criteria set as $1 \times 10-$ 6 applying Pulay's ${ }^{24}$ routine on SCF cycles. From single point calculations, orbital and gap energies and orbital densities were obtained. Time-Dependent Density Functional Theory (TD-DFT) ${ }^{25}$ was applied up to 6-EDOT to investigate excitations in gas phase with B3LYP/6-31+G level of theory for first 30 excited states for each EDOT geometry producing ultravioletvisible (UV-Vis) spectra for all geometries of interest (Chart 1). The discussions are centered on comparison between theoretical and experimental UV-Vis spectra results, the contribution of an orbital in particular for the excited state and how the oligomer size affect the UV-Vis spectra.

\section{Results and Discussion}

Optimized molecular geometries from 1-EDOT to 10EDOT are shown in Figure 2, they present good agreement for a polymeric planar chain. Other theoretical works showed similar results, where were calculated a dihedral angle between monomers close to 180 degrees $^{26,27}$. From PM6 semi-empirical methodology, the mean dihedral angle calculated for each oligomer is shown in Table 1. Such result shows that is possible to produce good results for conductive polymers applying semi-empirical methodology with much less computational cost comparing to Hartree-Fock (HF) or DFT theory levels, once the main objective of the semiempirical calculations is produce a start molecular geometries to investigate electronic property.

Table 1. Mean Dihedral Angle (in degrees) between monomers from 1-EDOT to 10-EDOT from PM6 semi-empirical methodology.

\begin{tabular}{|c|c|}
\hline Geometry & Mean Dihedral Angle \\
\hline 1-EDOT & -- \\
\hline 2-EDOT & 179.89 \\
\hline 3-EDOT & 179.61 \\
\hline 4-EDOT & 179.35 \\
\hline 5-EDOT & 179.19 \\
\hline 6-EDOT & 179.98 \\
\hline 7-EDOT & 179.06 \\
\hline 8-EDOT & 178.98 \\
\hline 9-EDOT & 179.07 \\
\hline 10-EDOT & 179.13 \\
\hline
\end{tabular}

Considering the good agreement produced by PM6 methodology for PEDOT oligomers geometry, a new electronic structure calculation was performed through DFT theory. The choice to produce molecular geometries by semi-empirical calculations, analyzing electronic structure through DFT theory was made considering low computational cost to produce optimized geometries, and better electronic description, respectively ${ }^{8,25}$. From 1-EDOT to 10-EDOT molecular structures (Figure 1) were calculated HOMO and LUMO orbitals, which are located over all oligomeric chain. It is possible to purpose that charge transport occur between sulfur atoms and $\pi$-type conjugated bonds over the neighbor monomers. The more acceptable electronic mechanism in solid state theory is based on excitation of the electrons from HOMO orbital to LUMO orbital. These electrons in LUMO orbital 
are available to perform the charge transfer or electronic conduction through oligomeric chain ${ }^{8,28}$. Such charge transport is often called charge way because the charge distribution is continuous in all molecular structure8. In fact, good results for PEDOT conductivity ${ }^{29}$ are explained by $\pi$ conjugation length as shown in Figure 1 .
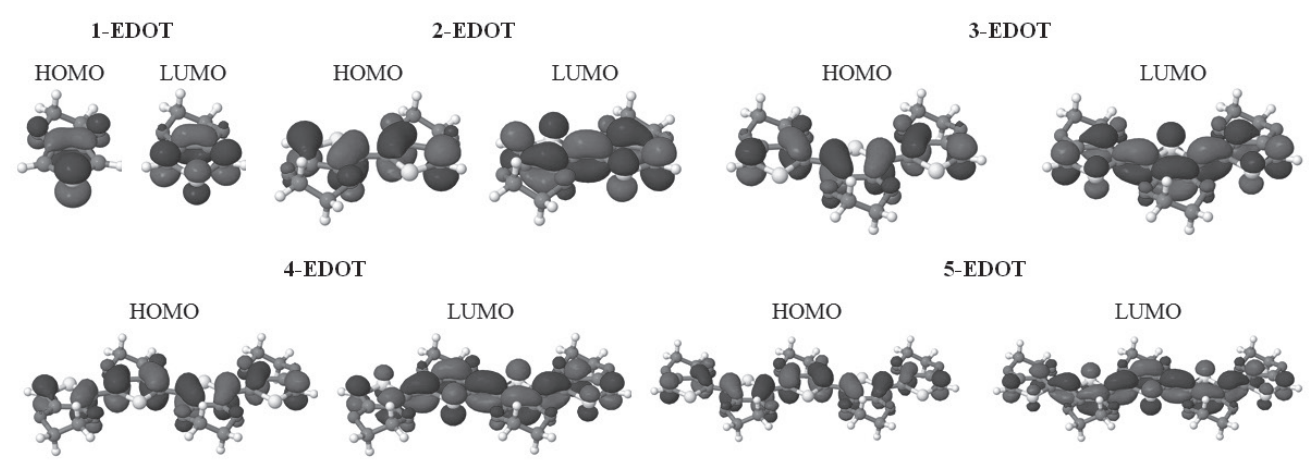

6-EDOT
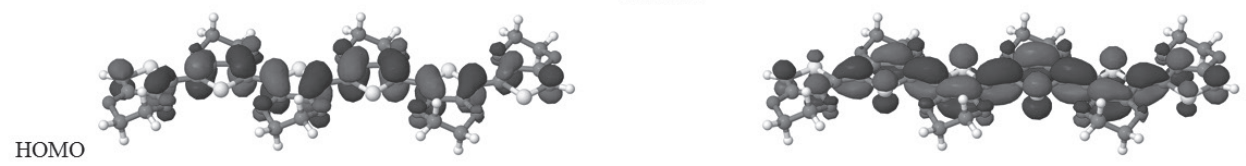

LUMO

7-EDOT

Hомо
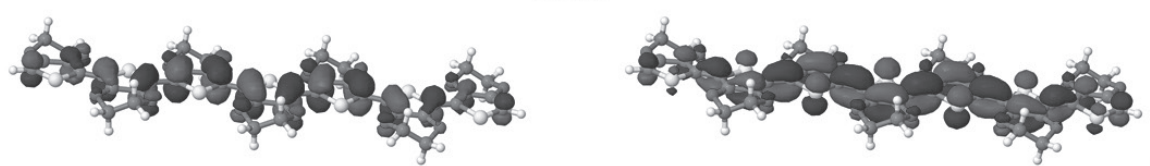

LUMO

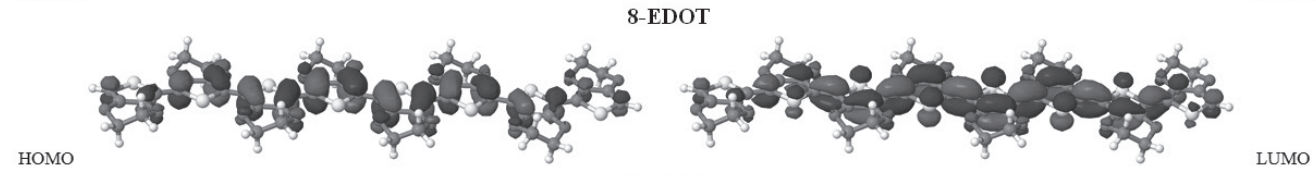
9-EDOT

LUMO

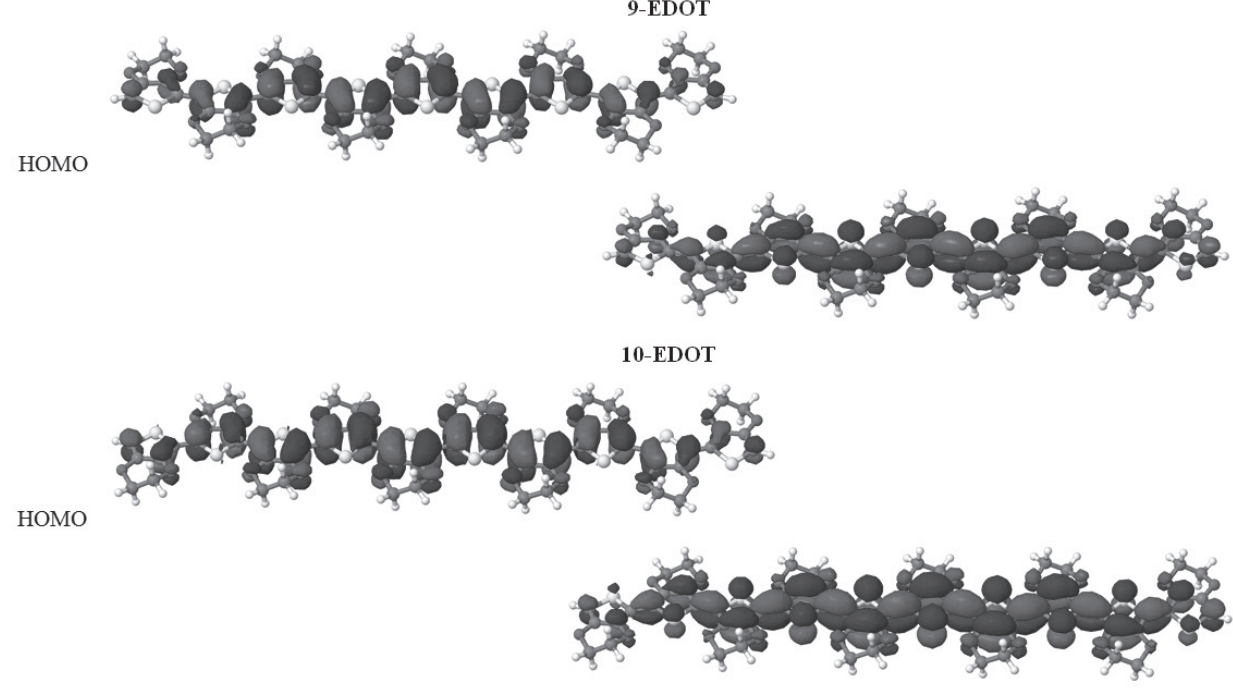

LUMO 
Potential Energy Surface (PES - Figure 2) results from 1-EDOT to 10-EDOT show that growth oligomeric chain cause bulk formation inside chain producing a charge way in center of the oligomer. This effect is only visualized from six monomeric units or 6-EDOT model. In the same context, these PES results provided information about why
PEDOT produces better results for electronic conduction in large oligomeric size than a few monomeric units, such as, 1-EDOT to 5-EDOT. Another point, for PEDOT macrostructure there is no intramolecular interaction becoming hard to perform charge transfer through $\pi-\pi$ stacking interaction among neighbor oligomeric chains.
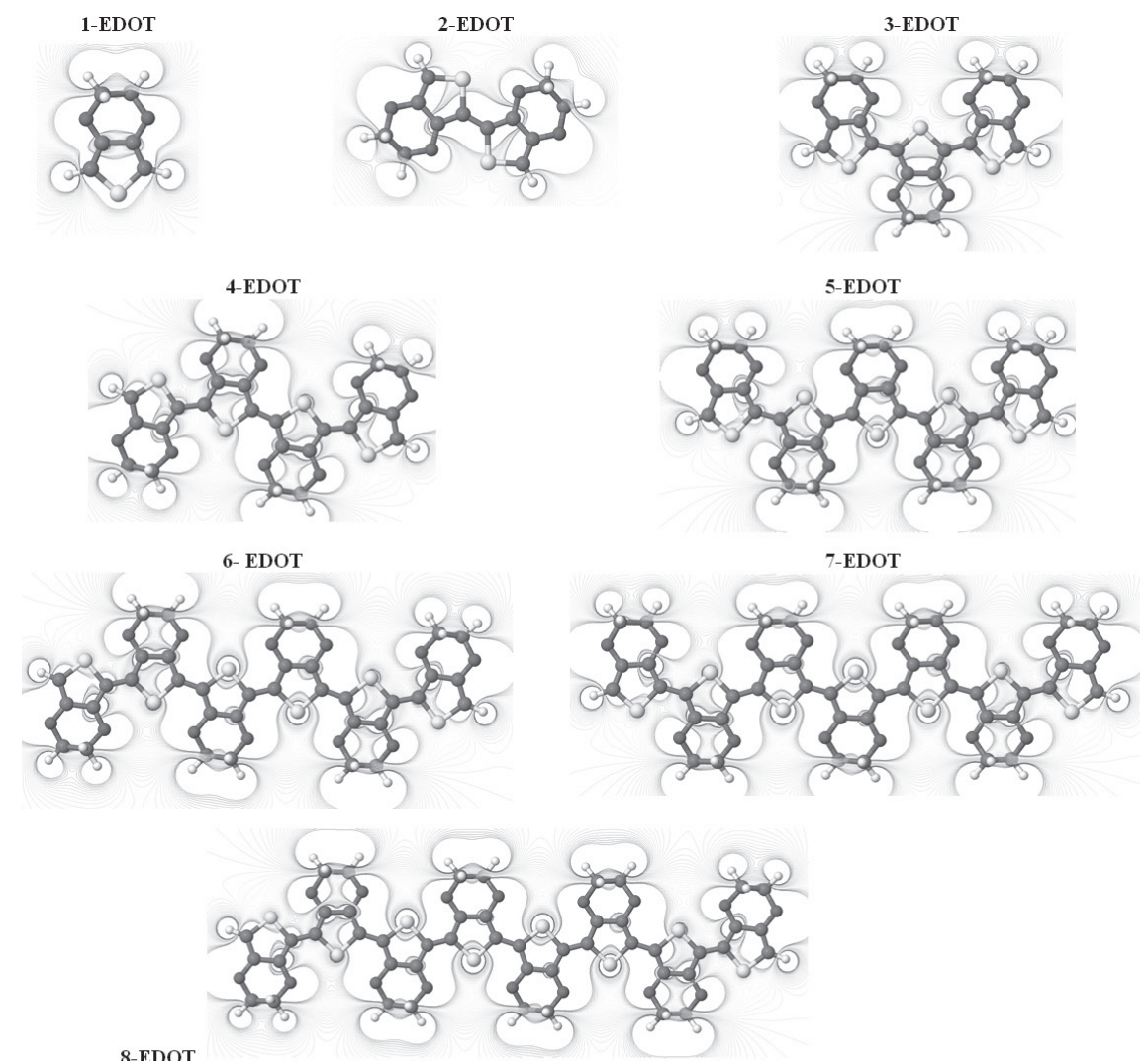

8-EDOT
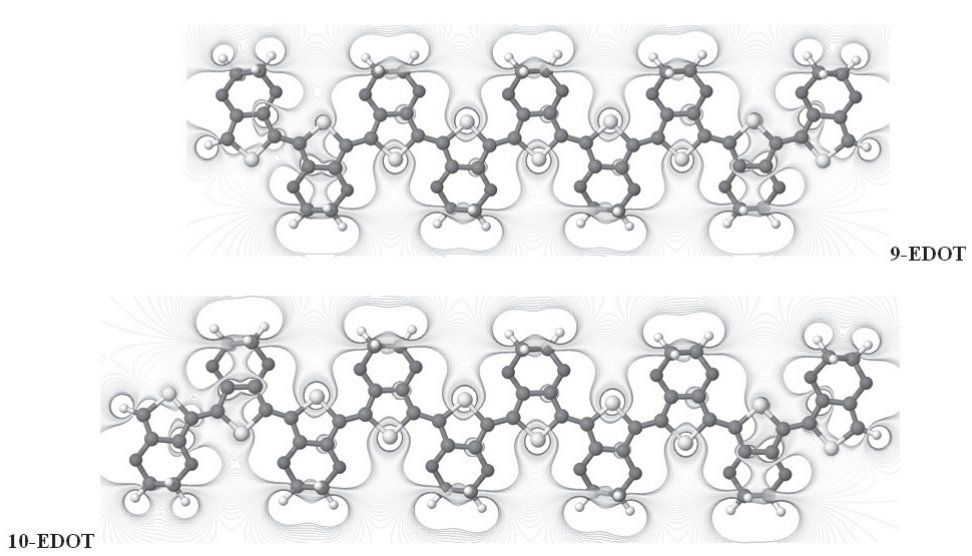

Figure 2. Potential energy surface (PES) calculated from 1-EDOT to 10-EDOT models using DFT/B3LYP 
Recently, TD-DFT have gained attention of computational chemists to provide information about specific excitation of frontiers orbitals. This approach has produced better understanding of electron-hole transfers from simulations based on Theory Orbital Molecular (TOM). Specially for organic semiconductors is possible to visualize where charge transport phenomenon is localized on molecular structure. Thus, TD-DFT simulations have represented better band-gap energy (Eg), PES and Density of States (DOS) results, mainly from calculations based on singlet excitation of the molecule. In our investigation, TD-DFT calculations from 1-EDOT to 6-EDOT electronic excitation have the dependence from oligomer size as shown in Figure 5. A red shift is observable as oligomeric size increases; however, from 7 monomeric units, TD-DFT calculations showed a high computationally cost and it was not possible to perform such calculations.

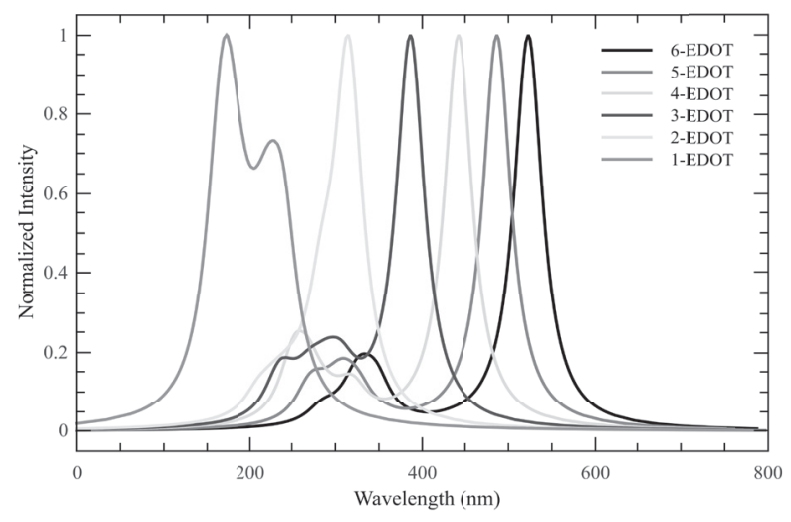

Figure 5. TD-DFT results for electronic excitation spectra from 1-EDOT to 6-EDOT molecules. Red shift is function of oligomer size.

Results for contribution of orbital excitation are shown in Table 2. Lower excitation energies are always associated to HOMO-LUMO excitation state for all simulated molecular structures. Furthermore, the HOMO-LUMO excitation energy or Eg is reduced from $5.18 \mathrm{eV}$ (1-EDOT) to $2.37 \mathrm{eV}$ (6-EDOT), showing a dependence of the $\mathrm{Eg}$ in relation to $\pi$ conjugation length. Other possibles excitation states from HOMO -2, HOMO -1 and LUMO +1 orbitals have also important contributions for excitation energy. For 1-EDOT molecule, the electronic excitation between HOMO - 1 to LUMO orbitals represents $93 \%$ of the total contribution for excitation in $5.57 \mathrm{eV}$; whereas, for 2-EDOT molecule, the same electronic excitation has $4.37 \mathrm{eV}$. However, two oscillator strength were produced with different contributions of $51 \%$ and $43 \%$ from HOMO -2 and HOMO
-1 orbitals to LUMO orbital, respectively. Oscillator strengths associated to such electronic transitions are equals to $0.05 \mathrm{a}$. u. and 0.07 a. u.; nevertheless, these oscillator strengths have opposite signals for contributions, suggesting that electronic excitation from both orbitals is possible. In the 4-EDOT oligomer, the excitation in $3.85 \mathrm{eV}$ is connected to HOMO -2 to LUMO electronic transition representing $44 \%$ of the total contribution of the excitation; whereas, in $4.61 \mathrm{eV}$ the total contribution is $40 \%$ of the excitation energy corresponding to HOMO -1 to LUMO +1 excitation. For 5-EDOT molecule there is a HOMO -3 to LUMO excitation in $3.78 \mathrm{eV}$, correspondent to $55 \%$ of excitation energy and a HOMO -1 to LUMO +1 contribution is associated to two important excitation energies, in $3.98 \mathrm{eV}$ and $4.11 \mathrm{eV}$. At last, 6-EDOT molecule shows an electronic transition between HOMO -1 and LUMO +1 orbitals with $34 \%$ of contribution for excitation energy in $3.58 \mathrm{eV}$. All these results suggest that HOMO and LUMO orbitals are the most important frontier orbitals because show the lowest energy excitation; however, HOMO -3, HOMO -2, HOMO -1 and LUMO +1 are also important for charge transport.

Table 2. Excitation Energy (E), Oscillator Strength (fosc), Contribution of the Main Configuration Orbital $(\mathrm{H}=\mathrm{HOMO}$ and $\mathrm{L}=\mathrm{LUMO})$ and States related to singlet excitation on different EDOT oligomers from TD-DFT.

\begin{tabular}{|c|c|c|c|c|}
\hline Geometry & $\mathrm{E}(\mathrm{eV})$ & $\begin{array}{l}\text { Fosc } \\
\text { (a. u.) }\end{array}$ & $\begin{array}{l}\text { Main } \\
\text { conf. }\end{array}$ & Contrib. \\
\hline \multirow[t]{2}{*}{ 1-EDOT } & 5.18 & 0.15 & $\mathrm{H} \rightarrow \mathrm{L}$ & $92 \%$ \\
\hline & 5.57 & 0.09 & $\mathrm{H}-1 \rightarrow \mathrm{L}$ & $93 \%$ \\
\hline \multirow[t]{5}{*}{ 2-EDOT } & 3.94 & 0.52 & $\mathrm{H} \rightarrow \mathrm{L}$ & $98 \%$ \\
\hline & 4.37 & 0.05 & $\mathrm{H}-2 \rightarrow \mathrm{L}$ & $51 \%$ \\
\hline & & & $\mathrm{H}-1 \rightarrow \mathrm{L}$ & $43 \%$ \\
\hline & 4.37 & 0.07 & $\mathrm{H}-1 \rightarrow \mathrm{L}$ & $51 \%$ \\
\hline & & & $\mathrm{H}-2 \rightarrow \mathrm{L}$ & $43 \%$ \\
\hline \multirow[t]{3}{*}{ 3-EDOT } & 3.21 & 0.88 & $\mathrm{H} \rightarrow \mathrm{L}$ & $99 \%$ \\
\hline & 4.06 & 0.07 & $\mathrm{H}-3 \rightarrow \mathrm{L}$ & $80 \%$ \\
\hline & 5.26 & 0.05 & $\mathrm{H}-3 \rightarrow \mathrm{L}+1$ & $63 \%$ \\
\hline \multirow[t]{3}{*}{ 4-EDOT } & 2.80 & 1.30 & $\mathrm{H} \rightarrow \mathrm{L}$ & $99 \%$ \\
\hline & 3.85 & 0.05 & $\mathrm{H}-2 \rightarrow \mathrm{L}$ & $44 \%$ \\
\hline & 4.61 & 0.06 & $\mathrm{H}-1 \rightarrow \mathrm{L}+1$ & $40 \%$ \\
\hline \multirow[t]{4}{*}{ 5-EDOT } & 2.55 & 1.70 & $\mathrm{H} \rightarrow \mathrm{L}$ & $99 \%$ \\
\hline & 3.78 & 0.07 & $\mathrm{H}-3 \rightarrow \mathrm{L}$ & $55 \%$ \\
\hline & 3.98 & 0.08 & $\mathrm{H}-1 \rightarrow \mathrm{L}+1$ & $50 \%$ \\
\hline & 4.11 & 0.08 & $\mathrm{H}-1 \rightarrow \mathrm{L}+1$ & $30 \%$ \\
\hline \multirow[t]{2}{*}{ 6-EDOT } & 2.37 & 2.08 & $\mathrm{H} \rightarrow \mathrm{L}$ & $99 \%$ \\
\hline & 3.58 & 0.19 & $\mathrm{H}-1 \rightarrow \mathrm{L}+1$ & $34 \%$ \\
\hline
\end{tabular}


In fact, experimental results30 show a second electronic excitation state more energetic than HOMO-LUMO excitation; however, the researchers do not confirm which molecular excitation produced such electronic absorption. Our TD-DFT single excitation calculations identify this electronic excitation as different transitions among HOMO -3, HOMO -2, HOMO -1 and LUMO + 1 orbitals; however, such electronic transitions confirmed are dependents from oligomer size simulated. Therefore, the amount of energy levels are fundamentals to rise this electronic transition of high energy. This result is consequence of the introduction of energy levels inside bandgap in according to Theory of Bands for solid state ${ }^{28}$.

\section{Conclusion}

The results showed in this work provided better understanding of charge transport properties in EDOT oligomers and PEDOT. From optimized geometries with low computational cost, electronic structure was determined from DFT calculations, showing bulk formation from 6 monomeric units extending for longer polymeric chains. The same calculations provided information about frontier orbital location, showing polymeric central region the most important for charge transport properties. DOS explains how band- gap energy was reduced from increase of monomeric units, showing $\pi$ conjugation dependence between electronic and geometric structures. The same relation was observed on singlet excitation theory applying TD-DFT calculations. These results are in good agreement with experimental data for excitation energy, becoming possible to understand the main excitation bands in UV-Vis spectra for EDOT oligomers and PEDOT and its relation with frontier orbitals.

\section{References}

1. IEA and OECD, (2011).

2. J.-P. Kleider, J. Alvarez, A. Brézard-Oudot, M.-E. Gueunier-Farret and O. Maslova, Sol. Energy Mater. Sol. Cells, 135, 8, (2015).

3. S. Hedström and P. Persson, J. Phys. Chem. C, 116, 26700, (2012).

4. I. Constantinou, T.-H. Lai, D. Zhao, E. D. Klump, J. J. Deininger, C. K. Lo, J. R. Reynolds and F. So, ACS Appl. Mater. Interfaces, 7, 4826, (2015).

5. M. Lanzi, E. Salatelli, T. Benelli, D. Caretti, L. Giorgini and F. P. DiNicola, J. Appl. Polym. Sci., 132, n/a, (2015).

6. F. Hermerschmidt, A. S. Kalogirou, J. Min, G. A. Zissimou, S. M. Tuladhar, T. Ameri, H. Faber, G. Itskos, S. A. Choulis, T. D. Anthopoulos, D. D. C. Bradley, J. Nelson, C. J. Brabec and P. A. Koutentis, J. Mater. Chem. C, 3, 2358, (2015).

7. M. Raïssi, L. Vignau, E. Cloutet and B. Ratier, Org. Electron., 21, 86, (2015).
8. V. Coropceanu, J. Cornil, D. A. da Silva Filho, Y. Olivier, R. Silbey and J.-L. Bredas, Chem. Rev., 107, 926, (2007).

9. A. Facchetti, Chem. Mater., 23, 733, (2011).

10. G. Tu, A. Bilge, S. Adamczyk, M. Forster, R. Heiderhoff, L. J. Balk, D. Mühlbacher, M. Morana, M. Koppe, M. C. Scharber, S. A. Choulis, C. J. Brabec and U. Scherf, Macromol. Rapid Commun., 28, 1781, (2007).

11. M. T. Dang, L. Hirsch, G. Wantz and J. D. Wuest, Chem. Rev., 113, 3734, (2013).

12. H. Nikoofard and M. Gholami, Comptes Rendus Chim., 17, 1034, (2014).

13. L. Wen, D.-C. Jeong, A. Javid, S. Kim, J.-D. Nam, C. Song and J. G. Han, Thin Solid Films, 587, 66, (2015).

14. S. Das, D. P. Chatterjee, R. Ghosh and A. K. Nandi, RSC Adv., 5, 20160, (2015).

15. J. Liu, S. Pathak, T. Stergiopoulos, T. Leijtens, K. Wojciechowski, S. Schumann, N. Kausch-Busies and H. J. Snaith, J. Phys. Chem. Lett., 6, 1666, (2015).

16. B. Park, M. Pazoki, K. Aitola, S. Jeong, E. M. J. Johansson, A. Hagfeldt and G. Boschloo, ACS Appl. Mater. Interfaces, 6, 2074, (2014).

17. J. J. Apperloo, L. 'Bert' Groenendaal, H. Verheyen, M. Jayakannan, R. A. J. Janssen, A. Dkhissi, D. Beljonne, R. Lazzaroni and J.-L. Brédas, Chem. - Eur. J., 8, 2384, (2002).

18. J. J. P. Stewart, J. Mol. Model., 13, 1173, (2007).

19. J. Stewart, (HTTP://OpenMOPAC.net).

20. R. N. Camp and H. F. King, J. Chem. Phys., 75, 268, (1981).

21. M. S. Gordon and M. W. Schmidt, pp. 75-83.

22. M. S. Gordon and M. W. Schmidt, (2005), pp. 1167-1189.

23. M. W. Schmidt, K. K. Baldridge, J. A. Boatz, S. T. Elbert, M. S. Gordon, J. H. Jensen, S. Koseki, N. Matsunaga, K. A. Nguyen, S. Su, T. L. Windus, M. Dupuis and J. A. Montgomery, J. Comput. Chem., 14, 1347, (1993).

24. P. Pulay, J. Comput. Chem., 3, 556, (1982).

25. P. Elliott, F. Furche and K. Burke, (2008), pp. 91-165.

26. J. A. Durães, D. A. da S. Filho, A. M. Ceschin, M. J. A. Sales and J. B. L. Martins, J. Mol. Model., 20, 1, (2014).

27. T. P. Kaloni, G. Schreckenbach and M. S. Freund, J. Phys. Chem. C, 119, 3979, (2015)

28. W. A. Harrison, (2005).

29. L. Groenendaal, F. Jonas, D. Freitag, H. Pielartzik and J. R. Reynolds, Adv. Mater., 12, 481, (2000).

30. D. Wasserberg, S. C. J. Meskers, R. A. J. Janssen, E. Mena-Osteritz and P. Bäuerle, J. Am. Chem. Soc., 128, 17007, (2006).

Ageo M. de Andrade*a, Luis H. Lacerda ${ }^{\text {, }}$ Renan A. P. Ribeiro a, Sergio R. de Lazaro, Alexandre C. Junior ${ }^{b}$

a Department of Chemistry, State University of Ponta Grossa, Av. General Carlos Cavalcanti, 4748, 84030-900, Ponta Grossa, PR, Brazil

${ }^{b}$ Department of Physics, State University of Ponta Grossa, Av. General Carlos Cavalcanti, 4748, 84030-900, Ponta Grossa, PR, Brazil

*E-mail: aageo25@gmail.com 